Received: 26 April 2017

Accepted: 30 November 2017

Published online: 14 December 2017

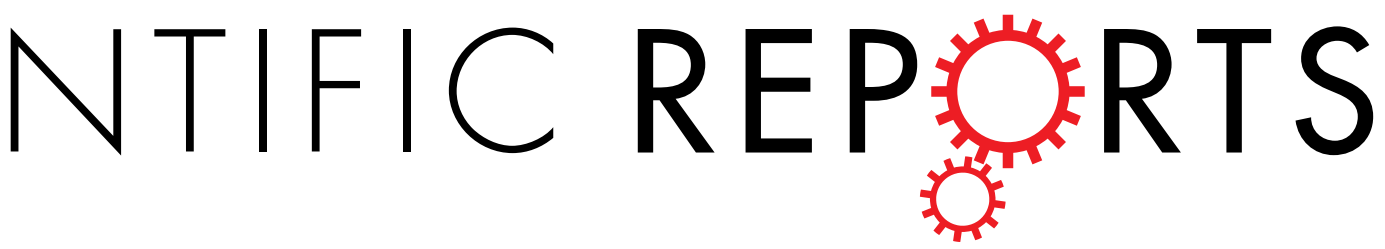

\title{
Surveillance of Live Poultry Markets for Low Pathogenic Avian Influenza Viruses in Guangxi Province, Southern China, from 2012-2015
}

Sisi Luo, Zhixun Xie, Zhiqin Xie, Liji Xie, Li Huang, Jiaoling Huang, Xianwen Deng, Tingting Zeng, Sheng Wang, Yanfang Zhang \& Jiabo Liu

Infections with low pathogenic avian influenza viruses (LPAIVs) can be mild or asymptomatic in poultry; however, in humans, LPAIVs can cause severe infections and death, as demonstrated by the H7N9 and H10N8 human infection outbreaks in 2013 in China. In this study, we conducted an epidemiological survey of LPAIVs at live poultry markets (LPMs) in Guangxi Province, Southern China, which is near several Southeast Asian countries. From January 2012 to December 2015, we collected 3,813 swab samples from poultry at LPMs in Guangxi. Viral isolation, hemagglutination inhibition assay and viral sequencing were utilized to identify LPAIVs in the collected samples. Among the samples, 622 (16.3\%) were positive for LPAIVs. Six subtypes $(\mathrm{H1}, \mathrm{H3}, \mathrm{H4}, \mathrm{H6}, \mathrm{H} 9$ and $\mathrm{H} 11)$ were individually isolated and identified. Of these subtypes, $\mathrm{H3}, \mathrm{H} 6$ and $\mathrm{H} 9$ were predominant in ducks, geese and chickens, respectively. Among the 622 positive samples, $160(25.7 \%)$ contained more than one subtype, and $\mathrm{H} 8, \mathrm{H} 10, \mathrm{H} 12, \mathrm{H} 13$, and $\mathrm{H} 16$ were identified among them, which highlights the continuous need for enhanced surveillance of AIVs. These results provide detailed information regarding the epidemic situation of LPAIVs in the area, which can aid efforts to prevent and control AIV transmission in humans and animals.

Avian influenza viruses (AIVs) are enveloped viruses of the genus Influenza A virus in the family Orthomyxoviridae, and their genome consists of eight segments of single-stranded, negative-sense RNA. AIVs are classified into distinct subtypes based on the antigenicity of their hemagglutinin (HA) and neuraminidase (NA) proteins. To date, $18 \mathrm{HA}$ subtypes (H1-H18) and 11 NA subtypes (N1-N11) have been identified. All of these subtypes were initially identified from avian species, with the exception of H17N10 and H18N11, which were recently found in bats ${ }^{1,2}$. According to Office International Des Epizooties (OIE) standards, AIVs are classified according to their level of virulence as either highly pathogenic AIVs (HPAIVs) or low pathogenic AIVs(LPAIVs) (http://www.oie.int/fileadmin/Home/eng/Health_standards/tahm/2.03.04_AI.pdf). A few H5 and H7 subtypes of AIV, such as H5N1 and H7N7, are virulent HPAIVs, leading to high morbidity and mortality in poultry. These subtypes are a public health security concern and cause severe economic losses in the poultry industry ${ }^{3-5}$. In contrast, LPAIVs strains, including the H1-H16 subtypes, typically cause mild disease or asymptomatic infections in poultry. Thus, LPAIVs have been largely neglected in global disease control programs. However, the genetic rearrangement and recombination of LPAIVs afford considerable opportunities for rapid and substantial viral changes through both antigenic shift and drift. Such changes may generate novel viruses with increased virulence that can pose substantial risks to poultry and human health.

In March 2013, H7N9, a novel influenza A virus causing human infection emerged in the Yangtze River Delta region with a fatality rate of approximately $30 \%$. H7N9 quickly spread to more than 18 provinces and municipalities in China ${ }^{6}$. According to the Disease Outbreak News issued by the World Health Organization (WHO) on September 5,2017, 1,558 laboratory-confirmed cases of human infection with H7N9 virus have been reported since early 2013 (http://www.who.int/csr/don/5-september-2017-ah7n9-china/en/). During the same period, another novel emergent virus, H10N8, was detected in Jiangxi Province, China, resulting in 3 human infections

Guangxi Key Laboratory of Veterinary Biotechnology, Guangxi Veterinary Research Institute, 51 You Ai North Road, Nanning, Guangxi, 530001, China. Correspondence and requests for materials should be addressed to Z.X. (email: xiezhixun@126.com) 


\begin{tabular}{|l|l|l|l|}
\hline \multirow{2}{*}{ Sample year } & \multicolumn{4}{|l|}{ LPAIV isolation rate (\%) Number of positive samples/total samples } \\
\cline { 2 - 4 } & Chickens & Ducks & Geese \\
\hline 2012 & $\mathbf{7 . 5}(14 / 187)$ & $\mathbf{9 . 3}(33 / 356)$ & $\mathbf{5 0 . 0}(18 / 36)$ \\
\hline 2013 & $\mathbf{2 1 . 6}(52 / 241)$ & $\mathbf{2 2 . 0}(95 / 431)$ & $\mathbf{3 4 . 8}(16 / 46)$ \\
\hline 2014 & $\mathbf{1 4 . 8}(61 / 413)$ & $\mathbf{1 5 . 8}(117 / 740)$ & $\mathbf{9 . 8}(11 / 112)$ \\
\hline 2015 & $\mathbf{8 . 0}(34 / 426)$ & $\mathbf{2 0 . 8}(157 / 753)$ & $\mathbf{1 9 . 4}(14 / 72)$ \\
\hline Total (2012-2015) & $\mathbf{1 2 . 7}(161 / 1,267)$ & $\mathbf{1 7 . 6}(402 / 2,280)$ & $\mathbf{2 2 . 2}(59 / 266)$ \\
\hline Grand total (all poultry samples 2012-2015) & $\mathbf{1 6 . 3 ( 6 2 2 / 3 , 8 1 3 )}$ & & \\
\hline
\end{tabular}

Table 1. LPAIVs isolated from chickens, ducks and geese from live poultry markets (LPMs) in Guangxi, Southern China, from January 2012 to December 2015.

and 2 deaths in December 20137. Nevertheless, both the H7N9 and H10N8 strains showed few symptoms and caused silent out breaks in poultry and were therefore classified as LPAIVs; however, these strains retain the ability to infect humans and cause death. Unlike HPAIV outbreaks that are direct and obvious, LPAIVs, such as H7N9 and H10N8, are harder to detect in poultry until they cross the species barrier and directly infect humans. When reports of human deaths due to LPAIVs emerge, people stop purchasing live poultry in live poultry markets (LPMs), which results in large numbers of poultry products (e.g., chickens, ducks, geese and eggs) that are unable to be sold, thereby causing serious economic losses in the poultry industry. In addition, the genetic reassortment of LPAIVs is cause for concern. The recently emerged H7N9 and H10N8 AIVs are reassortant viruses, and the H9N2 LPAIV contributed six internal genes to the novel H7N9 and H10N8 viruses ${ }^{8,9}$. A naturally acquired human infection with the H6N1 virus was first documented in Taiwan in June 2013. Sequence analyses reveal that the human isolate (A/Taiwan/2/2013, human-H6N1) from the patient was highly homologous to chicken H6N1 virus isolates in Taiwan ${ }^{10}$. LPAIVs are closely related to human health and pose potential threats to public health security.

Southern China has been considered an influenza epicenter due to its favorable breeding grounds for the virus. Domestic poultry farming in Southern China occurs in high-density settings and in a free-range manner. Waterfowl, especially ducks and geese, occupy lakes and other abundant water resources in Guangxi, Southern China. These settings create environments where migratory birds and waterfowl are in close contact, sharing water, food, and habitat, thus contributing to the geographical spread of AIVs via long-distance migration through flyways. Thus, waterfowl species play an important role in AIV transmission and are regarded as a natural reservoir of AIVs ${ }^{11}$. Guangxi Province borders Vietnam, where complex epidemics of AIVs, including H5N1, continue to occur ${ }^{12}$. Furthermore, trade in LPMs, a traditional practice in this area, is considered a major source of AIV dissemination. In LPMs, birds from different sources can be housed together for several days, providing opportunities for influenza virus reassortment and the cross-species transfer of AIV s ${ }^{13,14}$. In LPMs, humans interact closely with poultry and potentially share influenza pathogens, which can result in the emergence of novel AIV variants.

Little is known about the infection landscape of LPAIVs in Guangxi. Our comprehensive investigation of LPAIV infection patterns and distribution in LPMs in this region from 2009 to 2011 revealed that the epidemiology of LPAIVs in poultry in Guangxi is complex, highlighting the need for further epidemiological study ${ }^{15}$. In the present work, we report on continuous surveillance data from January 2012 through December 2015 involving a relatively sizeable collection of swab samples from the oropharyngeal and cloacal regions of chickens, ducks, and geese from LPMs in Guangxi. We examined these samples for the presence of LPAIVs (except for H5 and H7 LPAIVs). The resulting epidemiological data offer novel insights for planning strategies to prevent LPAIV infections in humans and other animals.

\section{Results}

Isolation rates of LPAIVs in chickens, ducks and geese. We collected 3,813 swabs samples from 21 different LPMs in Guangxi Province from January 2012 to December 2015. The samples were used for the isolation and identification of LPAIVs. As shown in Table 1, among the 3,813 samples, 622 were identified as positive for LPAIVs, including 161 from chickens, 402 from ducks and 59 from geese. The total isolation rate was $16.3 \%$ $(622 / 3,813$, positive/sum), and the isolation rates in chickens, ducks and geese were $12.7 \%(161 / 1,267), 17.6 \%$ $(402 / 2,280)$ and $22.2 \%$ (59/266), respectively. The rate of LPAIVs isolation was the highest from geese, followed by ducks and chickens, indicating that the isolation rate was higher in waterfowl. The numbers of positive samples and collected samples corresponding to the yearly isolation rates in chickens, ducks and geese from 2012 to 2015 are presented in Table 1. The isolation rate in ducks was slightly higher than the rate in chickens in 2012-2014 and was much higher than the rate in chickens in 2015. Compared with 2012, the isolation rates in both chickens and ducks increased to more than $20 \%$ in 2013 and then decreased to approximately $15 \%$ in 2014 . The isolation rate of LPAIVs in chickens continued to decrease thereafter, reaching $8 \%$ in 2015 , while that in ducks increased to $20.8 \%$. The isolation rate in geese was highest (50.0\%) in 2012. The isolation rate in geese decreased to $34.8 \%$ in 2013 and then to $9.8 \%$ in 2014 , but it subsequently increased to $19.4 \%$ in 2015 .

The season average isolation rates in poultry, including chickens, ducks and geese, during the four years are shown in Fig. 1a. Higher isolation rates occurred in the spring and winter, and the isolation rates were relatively low in summer and autumn. 

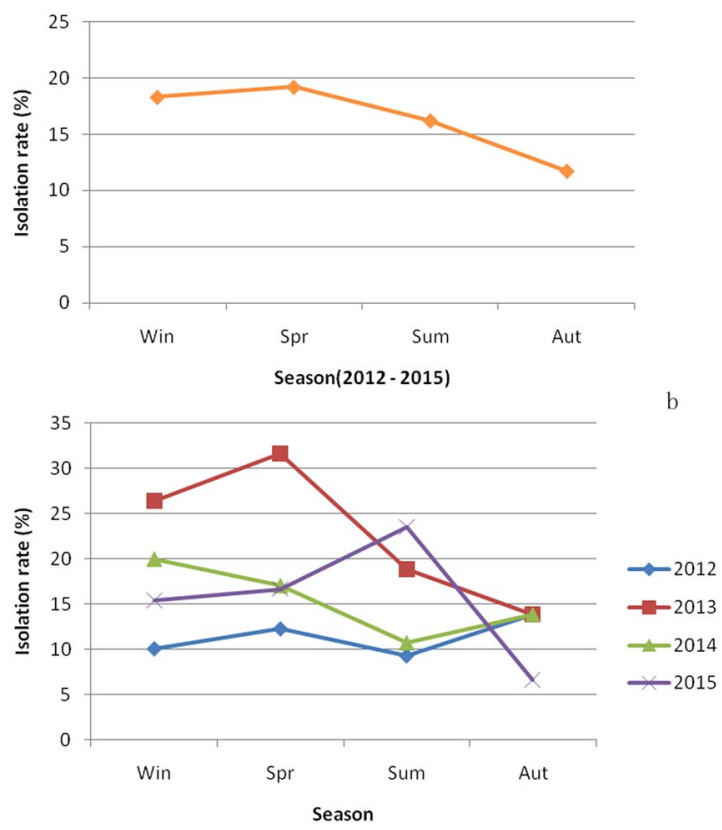

Figure 1. Relationship between season and isolation rates of LPAIVs in poultry from LPMs in Guangxi, Southern China, from 2012-2015. (a) Average isolation rates of each season over four years. (b) Isolation rates of each season annually.

\begin{tabular}{|l|l|l|l|l|l|l|l|}
\hline \multirow{2}{*}{ Number } & \multirow{2}{*}{$\begin{array}{l}\text { Subtype of } \\
\text { infection }\end{array}$} & \multicolumn{4}{|l|}{ Number of cases } & $\begin{array}{l}\text { Percentages in } \\
\text { positive samples (\%) }\end{array}$ & $\begin{array}{l}\text { Percentages in } \\
\text { collected samples (\%) }\end{array}$ \\
\cline { 3 - 8 } & Chickens & Ducks & Geese & Total & 0.1 \\
\hline 1 & H1 & 0 & 4 & 0 & 4 & 0.64 & 2.8 \\
\hline 2 & H3 & 7 & $\mathbf{9 8}$ & 2 & 107 & 17.2 & 2.0 \\
\hline 3 & H4 & 5 & 69 & 4 & 78 & 12.5 & 3.2 \\
\hline 4 & H6 & 10 & 70 & $\mathbf{4 2}$ & 122 & 19.6 & $\mathbf{3 . 9}$ \\
\hline 5 & H9 & $\mathbf{9 9}$ & 47 & 4 & $\mathbf{1 5 0}$ & $\mathbf{2 4 . 1}$ & 0.026 \\
\hline 6 & H11 & 0 & 1 & 0 & 1 & 0.16 & 4.2 \\
\hline 7 & Mixed infections & 40 & 113 & 7 & 160 & 25.7 & \\
\hline Total & & 161 & 402 & 59 & 622 & & \\
\hline
\end{tabular}

Table 2. Number of infections of different HA subtypes of LPAIVs in chickens, ducks and geese.

The season isolation rates are shown in Fig. $1 \mathrm{~b}$ from 2012 to 2015. In general, the isolation rate in 2013 was higher than the rates in the other three years. The highest isolation rates from 2012 to 2015 were in autumn, spring, winter and summer, respectively. Generally, the isolation rates in spring and winter are higher than in summer and autumn, except for the rather high rates in autumn in 2012 and in summer in 2015.

HA and NA subtypes identified in chickens, ducks and geese. More than six HA subtypes (H1, H3, H4, H6, H9, H11 and some that were unknown) were individually isolated and identified in poultry. The percentages of identified subtypes from poultry in the 622 positive samples and in the 3,813 collected samples are listed in Table 2. When considering only single infections, H9 was the most commonly isolated subtype; its percentages in all positive samples $(24.1 \%, 150 / 622)$ and in collected samples $(3.9 \%, 150 / 3,813)$ were the highest for the isolation of any single subtype (Table 2). The H9, H3 and H6 subtypes were the predominant LPAIVs identified in chickens, ducks and geese, respectively. It is equally important that $78 \mathrm{H} 4$ isolates were identified, which were mainly from ducks.

The distribution of the HA subtypes in poultry is shown in Fig. 2. The H9 subtype was predominant in chickens, representing $61.5 \%$ of the isolates, with the remaining three subtypes each representing less than $7 \%$.The duck isolates were diverse, with six identified subtypes. H3 was the most frequent subtype (24.38\%), followed by H6 (17.41\%), H4 (17.16\%) and H9 (11.69\%). The least frequent were H1 and H11, which were isolated as single subtypes only in ducks. The goose isolates were less diverse than those of the ducks; the H6 subtype was predominant, with an isolation rate of $71.19 \%$, followed by $\mathrm{H} 4$ and $\mathrm{H} 9$, at equal rates of $6.78 \%$.

The $\mathrm{H} 1$ and $\mathrm{H} 11$ subtypes were sporadic, while the H3, H4, H6 and $\mathrm{H} 9$ subtypes can be isolated in any season. Figure 3 shows the relationship between the main isolation subtypes and season. The highest isolation rate 

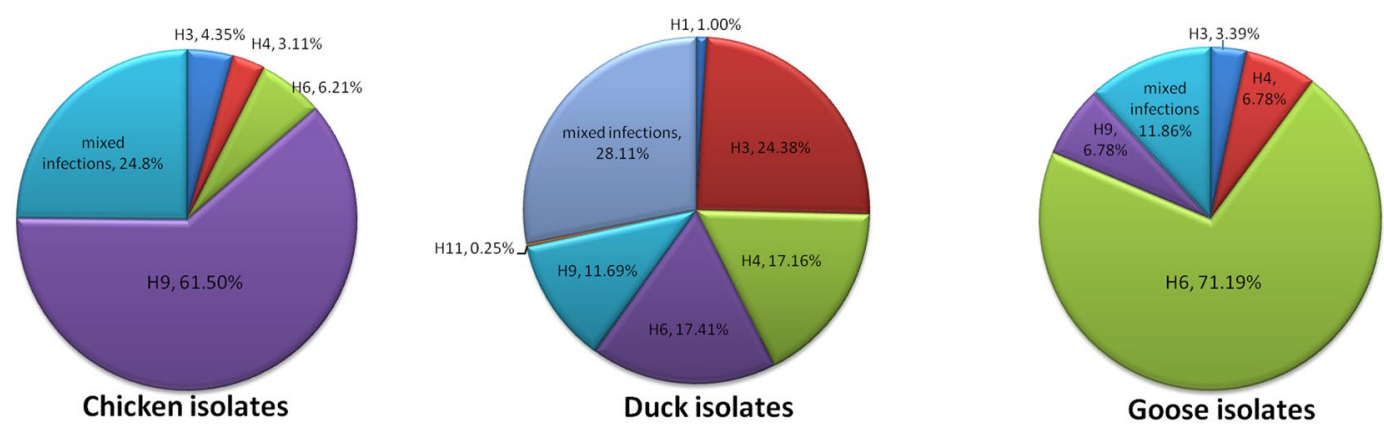

Figure 2. Distribution of HA subtypes among LPAIVs identified in poultry from LPMs in Guangxi, Southern China.

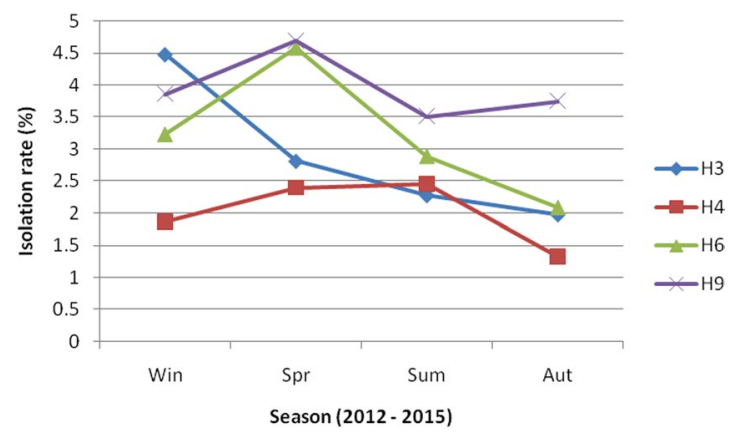

Figure 3. Relationship between season and isolation rates of the $\mathrm{H} 3, \mathrm{H} 4, \mathrm{H} 6$ and $\mathrm{H} 9$ subtypes in poultry from LPMs in Guangxi, Southern China, from 2012-2015.

\begin{tabular}{|l|l|l|l|l|l|l|}
\hline \multirow{2}{*}{ Combinations of mixed infection } & \multicolumn{4}{|l|}{ Number of cases } & $\begin{array}{l}\text { Percentages in mixed } \\
\text { infections (\%) }\end{array}$ & $\begin{array}{l}\text { Number of } \\
\text { Types }\end{array}$ \\
\cline { 2 - 8 } & Chickens & Ducks & Geese & Total & 17 \\
\hline Two different HA subtypes & 34 & 102 & 7 & 143 & 89.4 & 7 \\
\hline Three different HA subtypes & 6 & 6 & 0 & 12 & 7.5 & 2 \\
\hline Four different HA subtypes & 0 & 4 & 0 & 4 & 2.5 & 1 \\
\hline Six different HA subtypes & 0 & 1 & 0 & 1 & 0.6 & 27 \\
\hline Total & 40 & 113 & 7 & 160 & & 27 \\
\hline
\end{tabular}

Table 3. Number of mixed infections of different HA subtypes of LPAIVs in chickens, ducks and geese.

of the H3 subtype was in winter, and the H6 and H9 subtypes both peaked in spring. The HA and NA genes of the identified LPAIV isolates were sequenced and submitted to a BLAST search of the NCBI database. Three NA subtypes (N2, N6 and N8) were identified. The combinations of HA and NA subtypes identified in this study were H1N2, H3N2, H3N6, H4N2, H4N6, H4N8, H6N2, H6N6, H6N8, H9N2 and H11Nx (x has not been confirmed).

Mixed infections by different HA subtypes. The rate of mixed infections (more than one subtype) among the identified LPAIVs was $25.7 \%$ (160/622). Among the 160 samples positive for mixed infections, 40 were from chickens, 113 were from ducks, and 7 were from geese (Table 2). Among the 161 LPAIV-positive chicken isolates, 40 presented with mixed infections, yielding a rate of $24.84 \%$. The corresponding rates for ducks and geese were $28.11 \%$ and $11.86 \%$, respectively (Fig. 2). These results suggest that various mixed infections were widespread, especially in ducks. Ducks likely play an important role in the reassortment events that lead to new AIV variants.

As shown in Tables 3 and 4, among the mixed infections, combinations of two HA subtypes, comprising 17 types, were the most common combination type in the three hosts (chickens, ducks and geese), representing $89.4 \%$ of the mixed infections (143/160). Combinations of three HA subtypes, comprising 7 types, were observed only in chickens and ducks, and combinations of more than three HA subtypes, comprising 3 types, were observed only in ducks. These findings indicate that mixed infections in ducks were complex.

As shown in Table 4, the $\mathrm{H} 3+\mathrm{H} 4$ subtype was the most common combination of mixed infection, representing 48.8\% (78/160). Among the 78 strains of mixed infections with $\mathrm{H} 3+\mathrm{H} 4,57$ strains are from 2015, and 21 strains are from 2012-2014; therefore, the isolation of $\mathrm{H} 3+\mathrm{H} 4$ increased sharply in 2015. The different 


\begin{tabular}{|c|c|c|c|c|c|c|c|c|}
\hline \multirow[b]{2}{*}{ Number } & \multirow[b]{2}{*}{$\begin{array}{l}\text { Type of mixed infection } \\
\text { (27 combinations) }\end{array}$} & \multicolumn{7}{|c|}{ Number of cases } \\
\hline & & Chickens & Ducks & Geese & $\begin{array}{l}\text { Including H9 } \\
\text { (15 combinations) }\end{array}$ & \begin{tabular}{|l} 
Including $\mathrm{H4}$ \\
(15 combinations)
\end{tabular} & \begin{tabular}{|l} 
Including H3 \\
(8 combinations)
\end{tabular} & \begin{tabular}{|l} 
Including H6 \\
(8 combinations)
\end{tabular} \\
\hline 1 & $\mathrm{H} 1+\mathrm{H} 4$ & 0 & 1 & 0 & & 1 & & \\
\hline 2 & $\mathrm{H} 1+\mathrm{H} 9$ & 2 & 0 & 0 & 2 & & & \\
\hline 3 & $\mathrm{H} 1+\mathrm{H} 13$ & 0 & 1 & 0 & & & & \\
\hline 4 & $\mathrm{H} 3+\mathrm{H} 4$ & 5 & 69 & 4 & & 78 & 78 & \\
\hline 5 & $\mathrm{H} 3+\mathrm{H} 9$ & 1 & 2 & 0 & 3 & & 3 & \\
\hline 6 & H3 + H11 & 0 & 1 & 0 & & & 1 & \\
\hline 7 & $\mathrm{H} 4+\mathrm{H} 6$ & 0 & 4 & 0 & & 4 & & 4 \\
\hline 8 & $\mathrm{H} 4+\mathrm{H} 9$ & 11 & 11 & 0 & 22 & 22 & & \\
\hline 9 & $\mathrm{H} 4+\mathrm{H} 13$ & 0 & 5 & 0 & & 5 & & \\
\hline 10 & $\mathrm{H} 6+\mathrm{H} 9$ & 1 & 1 & 0 & 2 & & & 2 \\
\hline 11 & $\mathrm{H} 6+\mathrm{H} 11$ & 0 & 0 & 1 & & & & 1 \\
\hline 12 & $\mathrm{H} 6+\mathrm{H} 13$ & 2 & 4 & 1 & & & & 7 \\
\hline 13 & $\mathrm{H} 8+\mathrm{H} 9$ & 6 & 0 & 0 & 6 & & & \\
\hline 14 & H9+ H11 & 0 & 1 & 0 & 1 & & & \\
\hline 15 & $\mathrm{H} 9+\mathrm{H} 12$ & 4 & 1 & 1 & 6 & & & \\
\hline 16 & $\mathrm{H} 9+\mathrm{H} 13$ & 2 & 0 & 0 & 2 & & & \\
\hline 17 & $\mathrm{H} 9+\mathrm{H} 16$ & 0 & 1 & 0 & 1 & & & \\
\hline 18 & $\mathrm{H} 3+\mathrm{H} 4+\mathrm{H} 6$ & 0 & 1 & 0 & & 1 & 1 & 1 \\
\hline 19 & $\mathrm{H} 3+\mathrm{H} 4+\mathrm{H} 9$ & 0 & 1 & 0 & 1 & 1 & 1 & \\
\hline 20 & $\mathrm{H} 3+\mathrm{H} 4+\mathrm{H} 11$ & 0 & 1 & 0 & & 1 & 1 & \\
\hline 21 & $\mathrm{H} 4+\mathrm{H} 6+\mathrm{H} 9$ & 1 & 0 & 0 & 1 & 1 & & 1 \\
\hline 22 & $\mathrm{H} 4+\mathrm{H} 6+\mathrm{H} 13$ & 1 & 1 & 0 & & 2 & & 2 \\
\hline 23 & $\mathrm{H} 4+\mathrm{H} 8+\mathrm{H} 9$ & 3 & 2 & 0 & 5 & 5 & & \\
\hline 24 & $\mathrm{H} 4+\mathrm{H} 9+\mathrm{H} 16$ & 1 & 0 & 0 & 1 & 1 & & \\
\hline 25 & $\mathrm{H} 1+\mathrm{H} 4+\mathrm{H} 6+\mathrm{H} 10$ & 0 & 2 & 0 & & 2 & & 2 \\
\hline 26 & $\mathrm{H} 3+\mathrm{H} 4+\mathrm{H} 9+\mathrm{H} 11$ & 0 & 2 & 0 & 2 & 2 & 2 & \\
\hline 27 & $\mathrm{H} 3+\mathrm{H} 4+\mathrm{H} 9+\mathrm{H} 11+\mathrm{H} 13+\mathrm{H} 16$ & 0 & 1 & 0 & 1 & 1 & 1 & \\
\hline Total & & 40 & 113 & 7 & 56 & 127 & 88 & 20 \\
\hline The infection rate & & & & & $35.0 \%(56 / 160)$ & $79.4 \%(127 / 160)$ & $55.0 \%(88 / 160)$ & $12.5 \%(20 / 160)$ \\
\hline
\end{tabular}

Table 4. Mixed infections of different HA subtypes of LPAIVs in chickens, ducks and geese.

HA subtype combinations yielded 27 different types of mixed infections. Among these 27 types, there were 15 combinations involving the $\mathrm{H} 4$ subtype, totaling 127 strains, and $79.4 \%(127 / 160)$ among the mixed infections. Notably, mixed infections of 3-6 HA subtypes all involved the H4 subtype, indicating that the H4 subtype plays a significant role in mixed infections. H9 subtype distributed in the 15 combinations and 56 strains, $\mathrm{H} 3$ subtype distributed in the 8 combinations and 88 strains, and H6 subtype distributed in the 8 combinations and 20 strains, suggesting that the $\mathrm{H} 9, \mathrm{H} 3$ and $\mathrm{H} 6$ subtypes were also important components in mixed infections. Generally, $\mathrm{H} 4$ was the most abundant subtype in mixed infections. The number of combinations and the infection rate of the $\mathrm{H} 9$ subtype were both higher than those for H6 but lower than those for the H4 subtype. The rate of mixed infection cases including $\mathrm{H} 3$ was higher than that including $\mathrm{H} 9$, but only distributed in the 8 combinations, which was lower than those for the $\mathrm{H} 9$ ( 15 combinations). The high rates of cases that included $\mathrm{H} 3$ or $\mathrm{H} 4$ were mainly due to high proportion of $\mathrm{H} 3+\mathrm{H} 4$ in the mixed infection cases. In addition, $\mathrm{H} 8, \mathrm{H} 10, \mathrm{H} 12, \mathrm{H} 13$, and $\mathrm{H} 16$ were rare subtypes that were not isolated or identified among the single infections but were identified among the mixed infections.

\section{Discussion}

AIVs are distributed worldwide, with a global impact on animal and human health. When a poultry bird, such as a duck, is infected with multiple subtypes of AIV, the eight gene segments from the different HA subtypes may exchange genetic information with each other, which can lead to the emergence of a novel AIV and a potentially unpredictable pathogenic strain that could infect humans. The outbreak of the highly pathogenic H5N1 strain in 1997 was the first evidence that AIVs had the capacity for direct transmission from avian species to humans ${ }^{3}$. The 2009 pandemic H1N1 influenza virus spread to humans in over 215 countries and caused hundreds of thousands of deaths ${ }^{16}$. Previously, it was thought that only the $\mathrm{H} 5$ and $\mathrm{H} 7$ subtypes posed a significant pandemic risk; however, it is now known that strains of other HA subtypes (e.g., H9, H6 and H10) can infect humans and have pandemic potential ${ }^{17}$. LPAIV surveillance is intrinsically difficult because most LPAIV strains do not cause symptoms or only cause mild symptoms in infected poultry, allowing the viruses to spread silently ${ }^{18}$. To address this obstacle, intensive surveillance must be conducted if newly emerged reassortant viruses are to be detected early. 
Livestock breeding is extensive and booming in Guangxi, with many breeding farms and companies due to the subtropical climate. Many large-scale farms are located outside of the city and provide poultry production to meet strong and increasing consumer demand. Some of these products are sold to the adjacent Guangdong Province and Hong Kong. In some rural areas, chickens are freely raised on the mountainside, and ducks and geese range freely in pools, rivers and seaside areas. TheChina - ASEAN (The Association of Southeast Asian Nations) Exposition has convened in Nanning (the capital of Guangxi Province) annually since 2004, and people from different countries in Southeast Asia gather to discuss issues related to communication, culture and economy involved with the effective prevention and control of influenza viruses. The prevention and control of AIVs requires further attention and action in this region of the world. In addition, the seaside cities of Beihai and Fangcheng along the Beibu Gulf in Guangxi Province provide a good resting area for migrating wild birds. This area also has a humid environment, which may benefit the survival, growth and transmission of LPAIVs. To date, thirty cases of human H7N9 infection have been reported in Guangxi since the emergence of the first H7N9 human infection in early 2013. Among the thirty cases, there were three cases in 2014, no cases in 2015 and 2016, and 27 cases in 2017 (as of September 10) (http://www.gxhfpc.gov.cn/xzzc/2017/0221/34600.html and http:// www.gxhfpc.gov.cn/gzdt/bt/2017/0616/38510.html). As a result, LPMs were closed and sterilized, and people refrained from purchasing poultry products, resulting in severe economic losses to the poultry industry.

In contrast to the results of our previous study ${ }^{15}$, the current work revealed that $\mathrm{H} 9$, not $\mathrm{H} 3$, was the most prevalent subtype, consistent with the current epidemic situation in China ${ }^{17}$. H9 is currently in widespread circulation, occurring mostly in combination with the N2 subtype. However, we did isolate a strain of H9N8 in 2009 (GenBank number: KF768214). H9N2 viruses have been found to possess increased fitness to escape immunization pressure and undergo antigen evolution and drift in chickens, leading to the prevalence of new genotypes ${ }^{19}$. H9N2 has also been reported to reassort with several other subtypes, including H5N1, H6N1 and H6N2 ${ }^{17}$. Thus, H9N2 viruses are high on the list of candidates that could cause another human influenza pandemic. Presently, the H9N2 subtype is a primary focus of concern because it is the donor of six internal gene segments to the H7N9 and H10N8 novel reassortment avian influenza strains. It is well known that the H5N1 strain, which was responsible for the 1997 human influenza outbreak in Hong Kong, was found to contain internal genes derived from $\mathrm{H} 9 \mathrm{~N} 2^{20}$. The crucial role of $\mathrm{H} 9 \mathrm{~N} 2$ viruses at the animal-human interface might be due to its wide host range, adaptation in both poultry and mammals, and extensive gene reassortment ${ }^{17}$. Our epidemiological survey revealed that the $\mathrm{H} 9$ subtype was an important component and widely distributed in 15 combinations (total 27 combinations) of the mixed infections. Among the chicken isolates, H9 viruses were the major isolation subtype. In our previous study ${ }^{21}$, the results of a receptor-binding analysis revealed that 16 of 17 strains of H9 Guangxi isolates bound to both $\alpha-2,6$-linked glycans and $\alpha$-2,3-linked glycans; only 1 strain bound solely to $\alpha$-2,3-linked glycans. The binding of the human influenza virus receptor, Siaa2-6 Gal, suggests that chickens may serve as an intermediate host and may be the source of transmission of the influenza virus to humans.

In our previous research, H6N1, H6N2, H6N5, H6N6 and H6N8 and combinations of these five subtypes were identified during 2009-2011 15 .These five subtypes also circulated in Eastern China from 2002 to $2010^{22}$; however, in this study, only three H6 subtypes (H6N2, H6N6 and H6N8) were circulating in Guangxi Province between 2012 and 2015, with H6N2 and H6N6 being predominant. It was reported that the H6N2 and H6N6 viruses coexisted in LPMs in several provinces of Southern China between 2008 and 2011, and approximately 34\% of the H6 isolates derived from LPMs have acquired the ability to bind to the human-like receptor ${ }^{23}$. The evidence suggests that the H6 subtype was widespread and combined with different NA subtypes. The H6 subtypes were common among the goose and duck isolates in this study, especially the goose isolates, representing much as $71.19 \%$ of the identified LPAIVs from geese; the reason for this requires further exploration. Currently, H6 AIVs have worldwide distribution, and strains of the virus have been detected in various animal species, suggesting that H6 has a broader host range than other subtypes ${ }^{23}$. Our previous research ${ }^{24}$ suggested that H6 subtype AIVs could be directly transmitted from ducks to pigeons. Many reports have revealed that H6 viruses can infect and be efficiently transmitted among mice and ferrets ${ }^{25}$ and that they circulate extensively and reassort frequently ${ }^{24,26}$. In addition, a serum antibody positive for $\mathrm{H} 6$ viruses has been found in poultry workers ${ }^{27}$, and a further study indicated that $\mathrm{H} 6$ seropositivity in human specimens in Southern China was significantly higher than in northern China ${ }^{28}$.

Six LPAIV subtypes were individually isolated in ducks, suggesting that ducks are an important host. In the duck isolates, the isolation rate of the H3 subtype AIV was the highest of all the subtypes identified, which emphasizes the importance of enhancing the surveillance of waterfowl-originating AIVs. Most of the H3 isolates matched with $\mathrm{N} 2$, while a few matched with $\mathrm{N} 6$ or N8. H3, particularly the H3N8 virus, is highly adaptive since it is found in multiple avian and mammal hosts. H3N8, which was first isolated in Miami in 1963, is the major cause of equine influenza; however, H3N8 viruses have not yet been isolated from humans ${ }^{29}$. The H3N8 and $\mathrm{H} 3 \mathrm{~N} 2$ isolates from LPMs were reported to have a close relationship with the H5N8 HPAIV circulating in Korea and the United States, suggesting that H3-like AIVs may contribute internal genes to the highly pathogenic $\mathrm{H} 5 \mathrm{~N} 8$ viruses $^{30}$. In our previous research, a duck isolate named A/duck/Guangxi/175D12/2014(H3N6) ${ }^{31}$ showed reassortment events between $\mathrm{H} 3$ and the $\mathrm{H} 5 \mathrm{~N} 6$ and $\mathrm{H} 7 \mathrm{~N} 2$ influenza viruses. Genomic analyses of A/ pigeon/Guangxi/020 P/2009 (H3N6) ${ }^{32}$ and A/goose/Guangxi/020 G/2009(H3N8) ${ }^{33}$ suggested that these viruses have undergone extensive reassortment with different AIV subtypes. Dequan Yang et al. previously reported that H3N2 isolates from LPMs and poultry slaughterhouses in Shanghai had reassorted with other AIVs, especially the $\mathrm{H} 5$ and $\mathrm{H} 7$ subtypes, probably in pigeons, domestic ducks, and wild birds ${ }^{34}$. An H3N2 isolate from Anhui, which also showed the highest sequence homology to the H7 AIVs, was presumed to be a reassortant of $\mathrm{H} 3$ and $\mathrm{H} 7 \mathrm{AIVs}^{35}$. H3 is the most ubiquitous subtype and has a wide host range, including humans, pigs, horses, dogs, cats, seals, poultry, and wild aquatic birds ${ }^{29}$. It was reported that an $\mathrm{H} 3 \mathrm{~N} 2$ isolate from duck could acquire the potential to infect humans after multiple infections in a pig population ${ }^{36}$. H3 causes the seasonal influenza that is widely found in humans. These findings highlight the need for further investigation, epidemiological surveillance, and genetic and evolutionary studies of H3-subtype AIVs. 
H4 viruses also circulate widely throughout the world and are transmitted to mammalian hosts. Our study revealed that the isolation rate of $\mathrm{H} 4$ subtypes significantly increased from year to year. In the mixed infections, more than two subtypes included the $\mathrm{H} 4$ subtype, suggesting that $\mathrm{H} 4$ was highly infectious in combination with other AIV subtypes and had the advantage of increased potential for reassortment compared to other subtypes, thereby leading to the emergence and outbreak of novel subtype influenza viruses, which could threaten public health. We considered the $\mathrm{H} 4$ subtype worthy of particular research focus. We found that $\mathrm{H} 4$ viruses frequently matched with $\mathrm{N} 2$ and N6; however, some $\mathrm{H} 4 \mathrm{~N} 8$ viruses were also identified in our study. It was reported that $\mathrm{H} 4 \mathrm{~N} 8$ viruses isolated from shorebird contained a unique PB1 gene and caused severe respiratory disease in mice ${ }^{37}$. We chose some representative isolates of the $\mathrm{H} 4$ subtype from this study to experimentally infect specific-pathogen-free (SPF) chickens and did not observe clinical symptoms, which suggests low pathogenicity. Specific antibodies against H4-subtype AIVs were detected in sera from swine and from people working on a chicken farm ${ }^{38,39}$. Eight genes of H4N6 isolated from a duck farm were closely related to H4N6 viruses from LPMs in Shanghai, indicating a potential correlation between AIVs from LPMs and farms ${ }^{40}$. The H4 LPAIVs infected mice directly without prior adaptation ${ }^{40,41}$. The extensive reassortment of H4 AIVs is worrisome because it may produce hybrid viruses that can jump to humans and cause major public health issues, as occurred with the newly emerged H7N9 influenza virus ${ }^{18}$. Therefore, further investigations of the mechanisms of H4 AIV mutation and reassortment are important to prepare for potential pandemics.

Not only were the $\mathrm{H} 9, \mathrm{H} 6, \mathrm{H} 3$ and $\mathrm{H} 4$ the main subtypes in the single infections, but they were also important in the mixed infections. $\mathrm{H} 8, \mathrm{H} 10, \mathrm{H} 12, \mathrm{H} 13$ and $\mathrm{H} 16$ were also involved in mixed infections, but none of them have yet been isolated as a single infection. $\mathrm{H} 8, \mathrm{H} 12, \mathrm{H} 13$ and $\mathrm{H} 16$ were found infrequently compared with the other subtypes but emerged in mixed infections, revealing that they were active and in circulation in the area. These findings suggest a probable increasing trend and the need to monitor these subtypes. The H10 subtype existed in mixed infections and was not isolated alone in this survey; however, H10N8 has caused human deaths in Jiangxi Province in China. Epidemics of mixed infections are wide spread and complex, and the co-infection of different HA subtypes in the same bird presents a great risk of gene rearrangement, which can lead to novel subtypes of AIVs. Furthermore, mixed infections have increased viral diversity through reassortment between viruses from different sources.

In conclusion, we investigated the epidemiology of LPAIVs in poultry from LPMs in Guangxi Province, Southern China, an epicenter of avian flu. Our study demonstrates a high prevalence of LPAIVs in poultry in this area and highlights a need to further investigate the genetics and evolution of LPAIVs.

\section{Materials and Methods}

Ethics statement. The present study was approved and conducted in strict accordance with the recommendations in the guide for the care and use of routinely sampled animals in LPMs by the Animal Ethics Committee of the Guangxi Veterinary Research Institute. Biological samples were gently collected from healthy chickens, ducks and geese using aseptic cotton swabs. The birds were not anesthetized before sampling, and each sampled bird was observed for $30 \mathrm{~min}$ after sampling before being returned to its cage.

Sample collection. The epidemiological surveillance of LPAIVs in poultry was conducted at 21 randomly selected LPMs in Guangxi Province, Southern China. Among these LPMs, six were sampled continuously during the study period. We performed sampling once every week, and the six markets were sampled in turn. We collected 3,813 swab samples (an oropharyngeal swab and a cloacal swab from the same bird were placed into the same collection tube and counted as a single sample) from LPMs from January 2012 to December 2015. The swabs were maintained in $1 \mathrm{ml}$ of transport medium containing antibiotics at $4{ }^{\circ} \mathrm{C}$ until arrival at the laboratory. The cotton swabs were repeatedly cleaned, wiped and discarded. The sample solutions were stored at $-70^{\circ} \mathrm{C}$ for viral isolation.

Viral isolation and identification. Samples were thawed and centrifuged for $10 \mathrm{~min}$ at $3,000 \times \mathrm{g}$ at $4^{\circ} \mathrm{C}$, and $0.2 \mathrm{ml}$ of the supernatant from each sample was inoculated into the allantoic cavity of 9- to 11-day-old SPF embryonated chicken eggs (Beijing Merial Biology Company, Beijing, China). The inoculated chicken embryos were incubated at $35^{\circ} \mathrm{C}$ and observed daily. Allantoic fluid was harvested from the embryos that died within $120 \mathrm{~h}$ of inoculation. The chicken embryos that survived longer than $120 \mathrm{~h}$ post-inoculation were chilled at $4{ }^{\circ} \mathrm{C}$, and the allantoic fluid was then harvested. The harvested allantoic fluid samples were then tested for hemagglutination (HA) using $1 \%$ suspensions of chicken erythrocytes. The hemagglutination inhibition (HI) assay was conducted to determine the HA subtypes of the HA-positive samples. Briefly, the HA-positive allantoic fluid samples were tested with sera of different HA subtypes (H1-H4, H6, H8-H16), Newcastle disease virus (NDV) and Egg drop syndrome (EDS) to determine the HA subtypes. The isolates selected for sequence analysis were biologically cloned by at least three rounds of limiting dilution in embryonated SPF eggs. HA subtypes were identified using the HI test and by sequencing. NA subtypes were determined by direct sequencing. Experiments using LPAIVs were conducted in biosafety level 2 laboratory or negative pressure biosafety laboratory facilities.

Viral sequencing. Viral RNA was extracted from infected allantoic fluid using a Body Fluid Viral DNA/RNA Miniprep Kit (Axygen Biosciences, Hangzhou, China) according to the manufacturer's instructions. cDNA was synthesized from viral RNA by reverse transcription with the 12-bp primer 5'-AGCAAAAGCAGG- $3^{\prime}$. PCR was performed using specific primers as described in previous research to obtain the full-length HA and NA genes ${ }^{42}$. The PCR products were purified with the TaKaRa Agarose Gel DNA Purification Kit Ver. 2.0 (TaKaRa, Dalian, China) and sequenced by Invitrogen of Guangdong Co., Ltd. A BLAST search was performed to compare the confirmed sequences against the nucleotide sequences of all known HA and NA gene subtypes in the GenBank database, and the HA and NA subtypes of the isolates were determined and verified. 


\section{References}

1. Tong, S. et al. A distinct lineage of influenza A virus from bats. Proc. Natl. Acad. Sci. USA 109, 4269-4274, https://doi.org/10.1073/ pnas.1116200109 (2012).

2. Tong, S. et al. New world bats harbor diverse influenza A viruses. PLoS Pathog. 9, e1003657, https://doi.org/10.1371/journal. ppat.1003657 (2013).

3. Claas, E. C. et al. Human influenza A H5N1 virus related to a highly pathogenic avian influenza virus. Lancet3 51, 472-477, https:// doi.org/10.1016/S0140-6736(97)11212-0 (1998).

4. Puzelli, S. et al. Human infection with highly pathogenic A(H7N7) avian influenza virus, Italy, 2013. Emerg. Infect. Dis. 20, 1745-1749, https://doi.org/10.3201/eid2010.140512 (2014).

5. Bevins, S. N. et al. Widespread detection of highly pathogenic H5 influenza viruses in wild birds from the Pacific Flyway of the United States. Sci. Rep. 6, 28980, https://doi.org/10.1038/srep28980 (2016).

6. Su, S. et al. Epidemiology, evolution, and recent outbreaks of avian influenza virus in China. J. Virol. 89, 8671-8676, https://doi. org/10.1128/JVI.01034-15 (2015).

7. Qi, W. et al. Genesis of the novel human-infecting influenza $\mathrm{A}(\mathrm{H} 10 \mathrm{~N} 8)$ virus and potential genetic diversity of the virus in poultry, China. Euro. Surveill. 19, 24993558 (2014).

8. $\mathrm{Pu}$, J. et al. Evolution of the H9N2 influenza genotype that facilitated the genesis of the novel H7N9 virus. Proc. Natl. Acad. Sci. USA 112, 548-553, https://doi.org/10.1073/pnas.1422456112 (2015).

9. Chen, H. et al. Clinical and epidemiological characteristics of a fatal case of avian influenza A H10N8 virus infection: a descriptive study. Lancet 383, 714-721, https://doi.org/10.1016/S0140-6736(14)60111-2 (2014).

10. Wei, S. H. et al. Human infection with avian influenza A H6N1 virus: an epidemiological analysis. Lancet Respir Med 1, 771-778, https://doi.org/10.1016/S2213-2600(13)70221-2 (2013).

11. Webster, R. G., Bean, W. J., Gorman, O. T., Chambers, T. M. \& Kawaoka, Y. Evolution and ecology of influenza A viruses. Microbiol. Rev. 56, 152-179 (1992).

12. Manabe, T. et al. Chronological, geographical, and seasonal trends of human cases of avian influenza A (H5N1) in Vietnam, 20032014: a spatial analysis. BMC Infect Dis 16, 64, https://doi.org/10.1186/s12879-016-1391-8 (2016).

13. Yuan, J. et al. Effect of live poultry market closure on avian influenza A(H7N9) virus activity in Guangzhou, China, 2014. Emerg. Infect. Dis. 21, 1784-1793, https://doi.org/10.3201/eid2110.150623 (2015).

14. Soares Magalhaes, R. J. et al. Live poultry trade in Southern China provinces and HPAIV H5N1 infection in humans and poultry: the role of Chinese New Year festivities. PLoS One 7, e49712, https://doi.org/10.1371/journal.pone.0049712 (2012).

15. Peng, Y. et al. Epidemiological surveillance of low pathogenic avian influenza virus (LPAIV) from poultry in Guangxi Province, Southern China. PLoS One 8, e77132, https://doi.org/10.1371/journal.pone.0077132 (2013).

16. Nelson, M. I. \& Vincent, A. L. Reverse zoonosis of influenza to swine: new perspectives on the human-animal interface. Trends Microbiol. 23, 142-153, https://doi.org/10.1016/j.tim.2014.12.002 (2015).

17. Sun, Y. \& Liu, J. H9N2 influenza virus in China: a cause of concern. Protein Cell 6, 18-25, https://doi.org/10.1007/s13238-014-0111-7 (2015).

18. Liang, L. et al. Genetics, receptor binding, replication, and mammalian transmission of $\mathrm{H} 4$ avian influenza viruses isolated from live poultry markets in China. J. Virol. 90, 1455-1469, https://doi.org/10.1128/JVI.02692-15 (2015).

19. Wei, Y. et al. Antigenic evolution of H9N2 chicken influenza viruses isolated in China during 2009-2013 and selection of a candidate vaccine strain with broad cross-reactivity. Vet. Microbiol. 182, 1-7, https://doi.org/10.1016/j.vetmic.2015.10.031 (2016)

20. Guan, Y., Shortridge, K. F., Krauss, S. \& Webster, R. G. Molecular characterization of H9N2 influenza viruses: were they the donors of the "internal" genes of H5N1 viruses in Hong Kong? Proc Natl Acad Sci USA 96, 9363-9367 (1999).

21. Xu, Q. et al. Isolation, Identification and Biological Characteristics of H9 Subtype Avian Influenza Viruses. Progress in Veterinary Medicine 37(9), 10-15(2016).

22. Zhao, G. et al. Molecular evolution of the H6 subtype influenza A viruses from poultry in eastern China from 2002 to 2010 . Virol. J. 8, 470, https://doi.org/10.1186/1743-422X-8-470 (2011).

23. Wang, G. et al. H6 influenza viruses pose a potential threat to human health. J. Virol. 88, 3953-3964, https://doi.org/10.1128/ JVI.03292-13 (2014).

24. Li, M. et al. Molecular characteristics of H6N6 influenza virus isolated from pigeons in Guangxi, Southern China. Genome Announc. 3, e01422-01415, https://doi.org/10.1128/genomeA.01422-15 (2015).

25. Gillim-Ross, L. et al. Avian influenza h6 viruses productively infect and cause illness in mice and ferrets. J. Virol. 82, 10854-10863, https://doi.org/10.1128/JVI.01206-08 (2008).

26. Yuan, R. et al. Reassortment of avian influenza A/H6N6 viruses from live poultry markets in Guangdong, China. Front. Microbiol. 7, 65, https://doi.org/10.3389/fmicb.2016.00065 (2016).

27. Yuan, J. et al. Origin and molecular characteristics of a novel 2013 avian influenza A(H6N1) virus causing human infection in Taiwan. Clin. Infect. Dis. 57, 1367-1368, https://doi.org/10.1093/cid/cit479 (2013).

28. Xin, L. et al. Seropositivity for avian influenza H6 virus among humans, China. Emerg. Infect. Dis. 21, 1267-1269, https://doi. org/10.3201/eid2107.150135 (2015).

29. Solorzano, A. et al. Cross-species infectivity of H3N8 influenza virus in an experimental infection in swine. J. Virol. 89, 11190-11202, https://doi.org/10.1128/JVI.01509-15 (2015).

30. Cui, H. et al. Phylogenetic analysis and pathogenicity of $\mathrm{H} 3$ subtype avian influenza viruses isolated from live poultry markets in China. Sci. Rep. 6, 27360, https://doi.org/10.1038/srep27360 (2016)

31. Liu, T. et al. Characterization of the whole-genome sequence of an H3N6 avian influenza virus, isolated from a domestic duck in Guangxi, Southern China. Genome Announc. 3, e01190-01115, https://doi.org/10.1128/genomeA.01190-15 (2015).

32. Liu, T. et al. Avian influenza virus with hemagglutinin-neuraminidase combination H3N6, isolated from a domestic pigeon in Guangxi, Southern China. Genome Announc. 3, e01537-01514, https://doi.org/10.1128/genomeA.01537-14 (2015).

33. Liu, T. et al. Genetic characterization of a natural reassortant H3N8 avian influenza virus isolated from domestic geese in Guangxi, Southern China. Genome Announc. 2, e00747-00714, https://doi.org/10.1128/genomeA.00747-14 (2014).

34. Yang, D. et al. Genetic analysis of H3N2 avian influenza viruses isolated from live poultry markets and poultry slaughterhouses in Shanghai, China in 2013. Virus Genes 51, 25-32, https://doi.org/10.1007/s11262-015-1198-5 (2015).

35. Li, C., Yu, M., Liu, L. \& Sun, H. Characterization of a novel H3N2 influenza virus isolated from domestic ducks in China. Virus Genes 52, 568-572, https://doi.org/10.1007/s11262-016-1323-0 (2016).

36. Shichinohe, S., Okamatsu, M., Sakoda, Y. \& Kida, H. Selection of H3 avian influenza viruses with SAalpha2,6Gal receptor specificity in pigs. Virology 444, 404-408, https://doi.org/10.1016/j.virol.2013.07.007 (2013).

37. Bui, V. N. et al. H4N8 subtype avian influenza virus isolated from shorebirds contains a unique PB1 gene and causes severe respiratory disease in mice. Virology 423, 77-88, https://doi.org/10.1016/j.virol.2011.11.019 (2012).

38. Ninomiya, A., Takada, A., Okazaki, K., Shortridge, K. F. \& Kida, H. Seroepidemiological evidence of avian H4, H5, and H9 influenza A virus transmission to pigs in southeastern China. Vet. Microbiol. 88, 107-114 (2002).

39. Kayali, G. et al. Evidence of infection with $\mathrm{H} 4$ and $\mathrm{H} 11$ avian influenza viruses among Lebanese chicken growers. PLoS One 6, e26818, https://doi.org/10.1371/journal.pone.0026818 (2011).

40. Shi, Y. et al. Characterizations of H4 avian influenza viruses isolated from ducks in live poultry markets and farm in Shanghai. Sci. Rep. 6, 37843, https://doi.org/10.1038/srep37843 (2016). 
41. Kang, H. M. et al. Genetic and antigenic characteristics of $\mathrm{H} 4$ subtype avian influenza viruses in Korea and their pathogenicity in quails, domestic ducks and mice. J. Gen. Virol. 94, 30-39, https://doi.org/10.1099/vir.0.046581-0 (2013).

42. Hoffmann, E., Stech, J., Guan, Y., Webster, R. G. \& Perez, D. R. Universal primer set for the full-length amplification of all influenza A viruses. Arch Virol 146, 2275-2289 (2001).

\section{Acknowledgements}

We thank Dr. Feng Li (South Dakota State University) for assistance with revising the manuscript. This study was supported by the Special Support Plan for National High-Level Talents (2016-37), the Guangxi Science Base and Talents Special Program (AD16380009) and the Research and Innovation Bridges Cooperation Program between China and England (2016YFE0124200) and the Guangxi Science Great Special Program (1222003-2-4).

\section{Author Contributions}

Designed the experiments and supervised the study: Z.X.X. Performed the experiments: S.S.L., Z.Q.X., L.J.X., L.H., J.L.H., X.W.D., T.T.Z., S.W., Y.F.Z. and J.B.L. Analyzed the data: S.S.L, Z.X.X, Z.Q.X. and L.J.X. Wrote the paper: S.S.L. Helped review the manuscript: Z.X.X.

\section{Additional Information}

Competing Interests: The authors declare that they have no competing interests.

Publisher's note: Springer Nature remains neutral with regard to jurisdictional claims in published maps and institutional affiliations.

(c) (i) Open Access This article is licensed under a Creative Commons Attribution 4.0 International

License, which permits use, sharing, adaptation, distribution and reproduction in any medium or format, as long as you give appropriate credit to the original author(s) and the source, provide a link to the Creative Commons license, and indicate if changes were made. The images or other third party material in this article are included in the article's Creative Commons license, unless indicated otherwise in a credit line to the material. If material is not included in the article's Creative Commons license and your intended use is not permitted by statutory regulation or exceeds the permitted use, you will need to obtain permission directly from the copyright holder. To view a copy of this license, visit http://creativecommons.org/licenses/by/4.0/.

(c) The Author(s) 2017 